\title{
LEFT VENTRICULAR EXTENSION INTO THE ABDOMEN
}

\author{
Abde Hakem SELEM ${ }^{\mathrm{A}}$ MD, \& Mohammed Shafee ${ }^{\mathrm{b}}$ MD. \\ ${ }^{a}$ Associate Professor, Department of Cardiology, Zagazig University. \\ ${ }^{\mathrm{b}}$ Radiologist, Ibn Sina faculty of medicine.
}

\begin{abstract}
A rare developmental anomaly of the heart is described. It consisted of a tube like extension of the left ventricular apex toward the umbilicus superficially under the anterior abdominal wall abutting anterior surface of the liver and contracting synchronously with the myocardium. It is associated with a membranous subaortic ventricular septal defect and a centrally localized heart in the anterior mediastinum (figure 1). Three similar cases were reported in the fifties of the last century. Although there were different associated anomalies with the other three cases but all four cases were similar in the presence of the elongated ventricle in a tube like structure superficially into the abdomen suggesting a similarity in development.

Keywords: Congenital heart disease, Left ventricular extension, Ventricular Septal defect.
\end{abstract}

\section{INRODUCTION}

O ur case is a male patient 6 years old, he is the first child of healthy Ethiopian family and has a healthy sister aged 2 years old. He was born after a normal pregnancy and was delivered normally. Few months after delivery, his mother was told that her baby has ventricular septal defect and he is in need for follow up. Recently, his mother came to our outpatient clinic complaining of pulsating mass in the epigastric area. Clinically, patient is an active child with normal weight and height for age $(113 \mathrm{~cm}$ height and $22 \mathrm{~kg}$ weight), pulse was $81 / \mathrm{m}$, and blood pressure in the arms was $100 / 80$. Apical impulses was palpated in the left and right $4^{\text {th }}$ intercostals space medial to the mid clavicular line with palpable thrill over the anterior chest wall (left and right parasternal area). By auscultation there is harsh systolic murmur grade $4 / 6$ all over the anterior chest wall propagating to the back in between the scapulae. A standard 12 lead electrocardiogram showed left axis deviation and deep $\mathrm{S}$ in $\mathrm{V} 1$ with poor progression of $\mathrm{R}$ wave in the anterior chest leads (figure 2), while the right chest leads showed regression of deep S that was observed in V1 and V2 (figure 3). X-ray chest posteroanterior view showed a centrally positioned heart (mesocardia) with double density of the right border of the heart (figure 4). Echocardiography detected the normal venoatrial, atrioventricular and ventriculoarterial concordance but the apex of the left ventricle was shifted medially. A subaortic membranous ventricular septal defect was observed measuring about $5 \mathrm{~mm}$ with left to right flow and maximum systolic pressure gradient of $77 \mathrm{mmHg}$ (figure 5, 6 and 7). We observed that the apex of the left ventricle was connected to a tube like structure that has synchronous pulsation with the left ventricle (figure 7). By changing the probe (using the vascular probe to detect a superficial structure) and following this pulsating cord it was extended superficially under the anterior abdominal wall from the apex of the heart up to near the umbilicus and ending in a rounded manner as a blunt pulsating tube (figure 8). The thickness of the wall of that tube was about $2 \mathrm{~mm}$ while its length is about $5 \mathrm{~cm}$. It has the same echogenisity and contractility like the myocardium and surrounded by a pericardium (figure 8, 9, 10 and 11). Blood flow velocity at the site of connection between this tube and the apex was about $1.1 \mathrm{~m} / \mathrm{sec}$, while it was about $0.5 \mathrm{~m} / \mathrm{sec}$ at the middle of that tube (figure 12).

MRI was done in a saggittal, axial and coronal plane for the patient. It confirmed the middle position of the heart in the center of the mediastinum and the presence of VSD. Also it showed the retrosternal extension of the LV apex downward under the anterior abdominal wall and touching the anterior surface of the liver (figure 13, 14 and 15).

Similar to this case there were three reported cases by Formijne ${ }^{1}$ (1950), Snellen et $\mathrm{al}^{2}$ (1952) and Clifford Parsons $^{3}$ (1958). All these three cases are similar to our case in the extension of the ventricular tissue from the apex of the heart (from either or both ventricles) toward the umbilicus in a trunk like prolongation through the diaphragm, in front of the liver. Also all these cases has a ventricular septal defect and a mesocardial position of the heart in the middle of the chest.

However, those three cases were associated with other congenital anomalies which were not present in our case. The case reported by Formijne $^{1}$ (1950) was 30-year-old woman who died during thoracotomy; there were persistence of two superior vena cavx, tricuspid atresia, a wide atrial septal defect and a rudimentary right ventricle. In the male infant described by Snellen et $\mathrm{al}^{2}$. (1952) there were an enlarged all four chambers but the right ventricle being larger than the left, and the pulmonary artery larger than the 
aorta. The case reported by Clifford Parsons ${ }^{3}$ (1958) there were tricuspid atresia and the right pulmonary vein was connected to the right atrium, big atrial septal defect (ostium secondum), hypertrophied left ventricle, diminutive right ventricle and hypoplastic pulmonary artery.

The similarity between these four cases is sufficient to suggest a common defect in development as it was suggested by Clifford Parsons $^{3}$ (1958). He explained the occurrence of this deformity in early embryonic life when heart develops in the neck and migrates to the thorax in close association with the septum transversum, which forms a diaphragm separating the heart from the abdominal cavity and the yolk sac. The separation becomes more marked when, in the

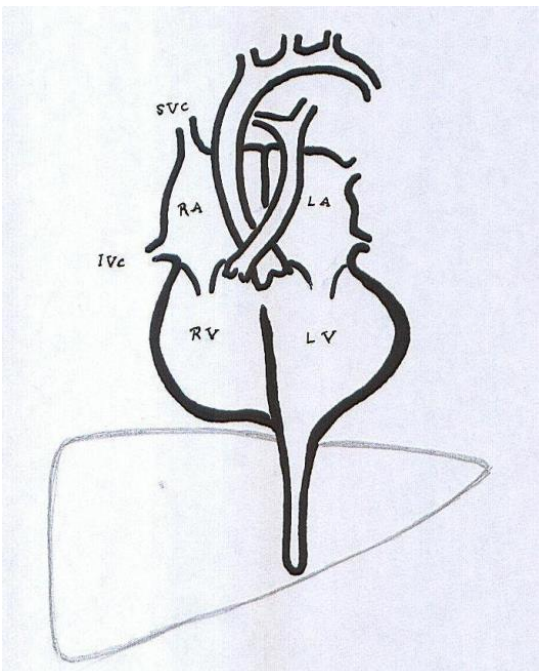

Figure 1 Diagrammatic representation of the anomaly.

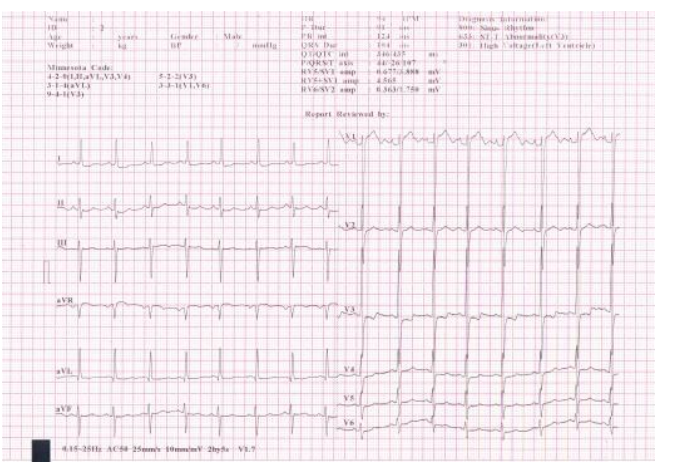

Figure 212 leads standard ECG. seventh week, the rapidly growing liver bulges forward and interposes between the heart and the stalk of the yolk sac. This elongated ventricular loop occurred if the developing heart being caught up in the structures of the yolk sac (umbilical structures) and it would be drawn out in front of the rapidly growing liver as the embryo straightened.

However, the therapeutic strategy that we suggest for this patient is to operate him surgically for closure of the VSD and removal of this ventricular extension because this superficially extended part of the left ventricle lying just under the anterior abdominal wall is liable to trauma and carry the risk of rupture and/or initiating severe arrhythmia

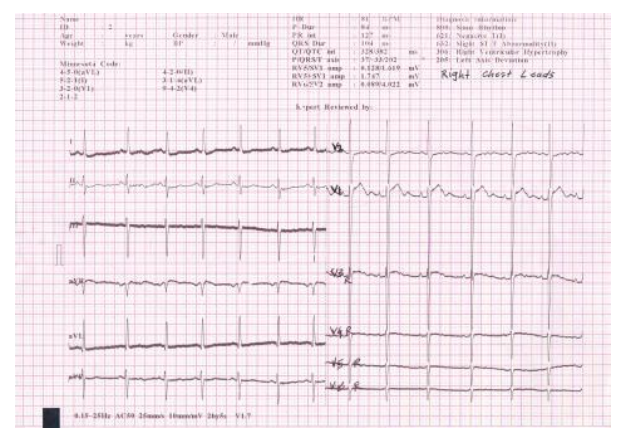

Figure 3 ECG with right chest leads.

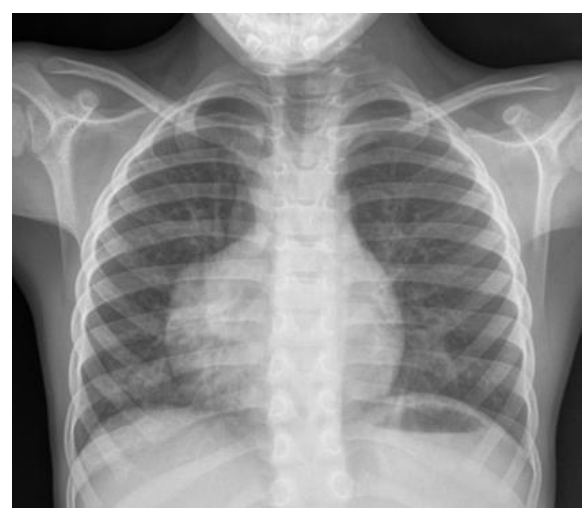

Figure 4, Posteroanterio $X$ ray chest. 


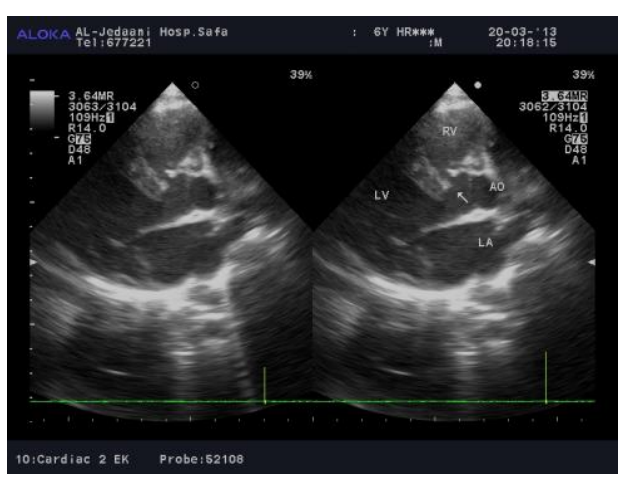

Figure 5 Parsternal view shoing the VSD.

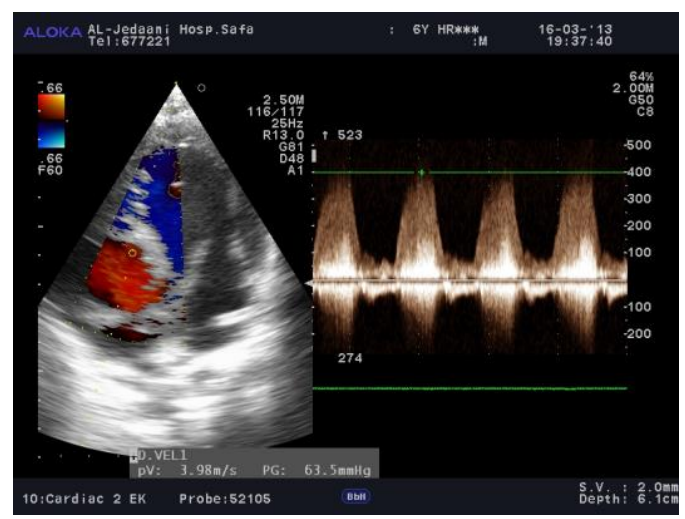

Figure 6 Parasternal short axis view to show the VSD flow.

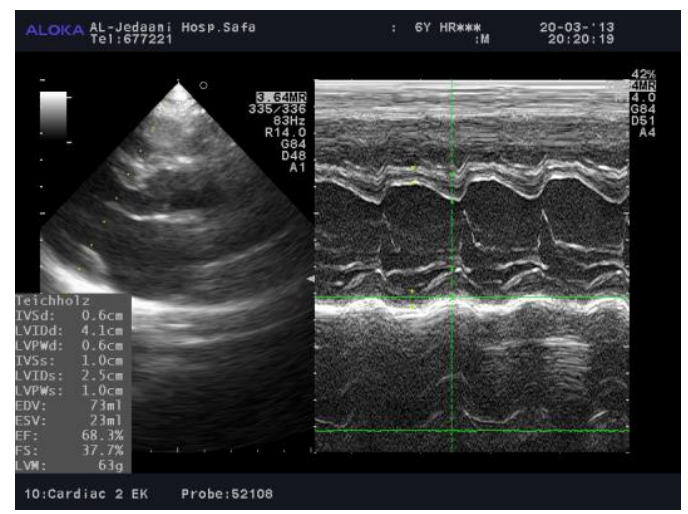

Figure $7 \mathrm{M}$ mode on the $\mathrm{LV}$ at the tips of the MV leaflets.

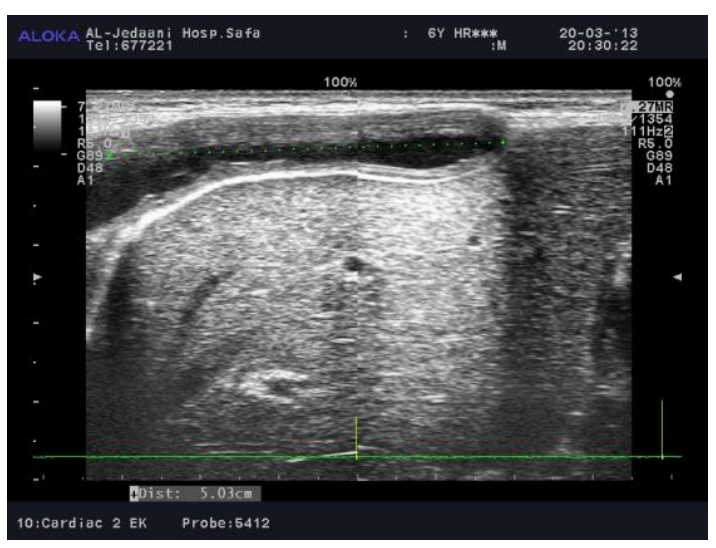

Figure 8 the length of the tube like extension of the $L V$ apex.

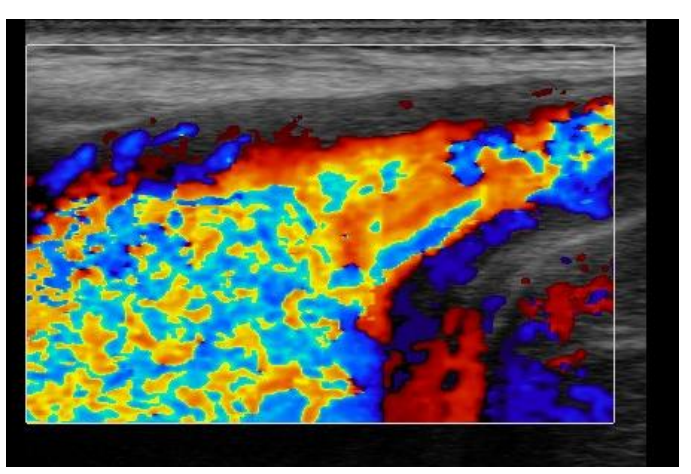

Figure 9 The apex of the $L V$ is connected to a tube like structure with blood flow inside.

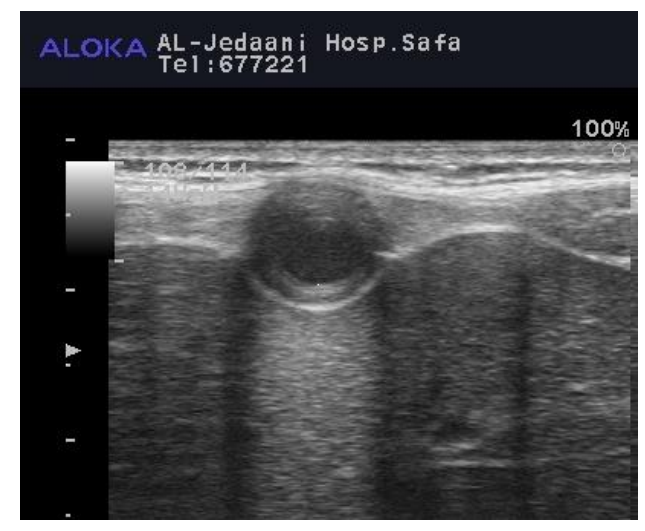

Figure 10 Short axis view on the tubal extension of the $L V$ infront of the liver. 


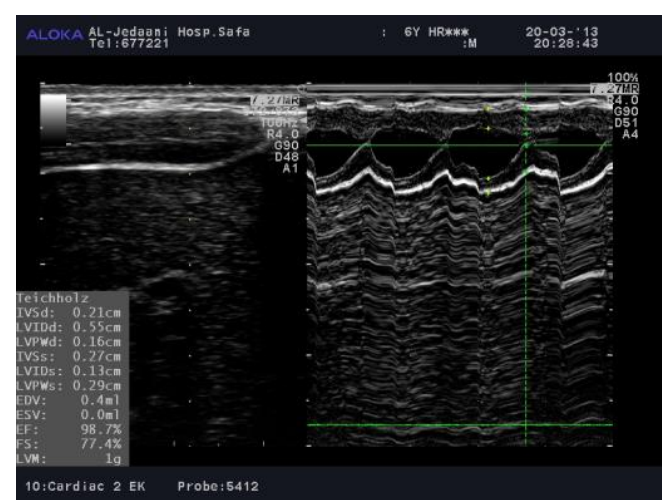

Figure $11 \mathrm{M}$ mode Echo on the tubal LV extension to show the contractility and thickness of the ventricular loop.

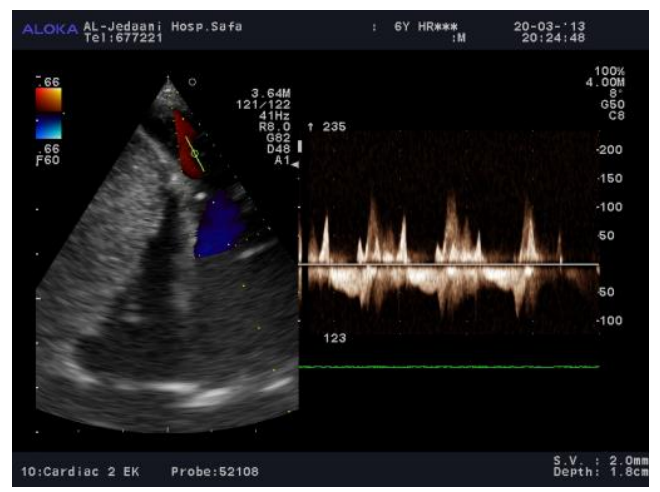

Figure 12 Blood flow velocity at the origin of the $\mathrm{LV}$ tubal extension.

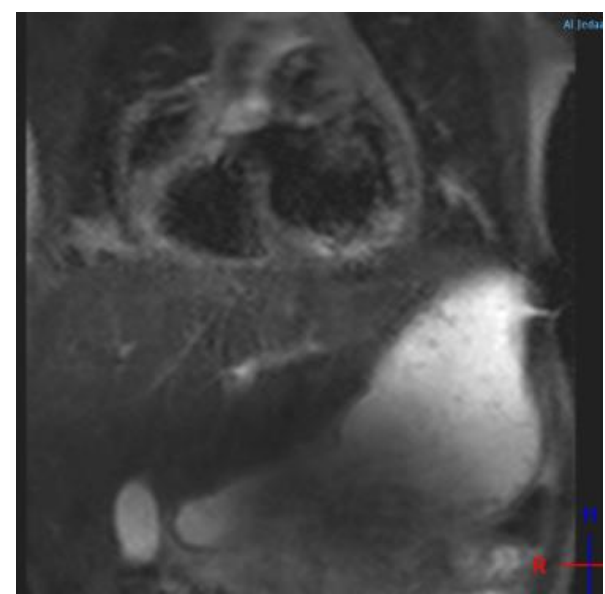

Figure 13 MRI coronal plane showing the VSD.

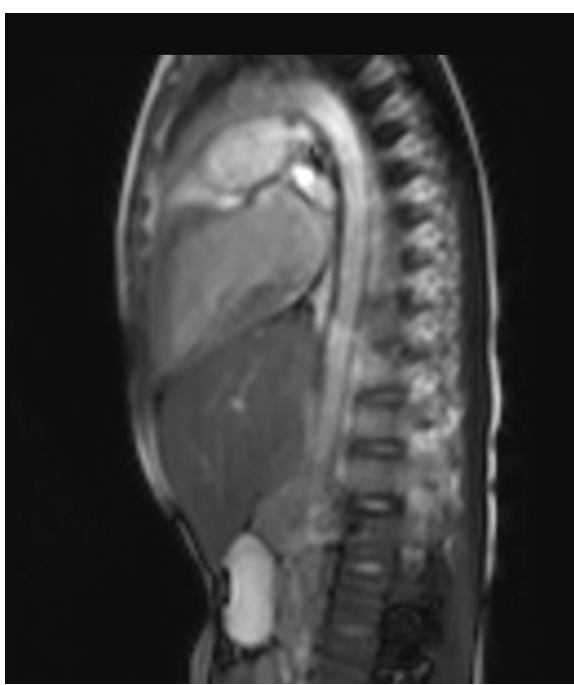

Figure 14, MRI Sagittal plane showing superficial extension of the heart in front of the liver.

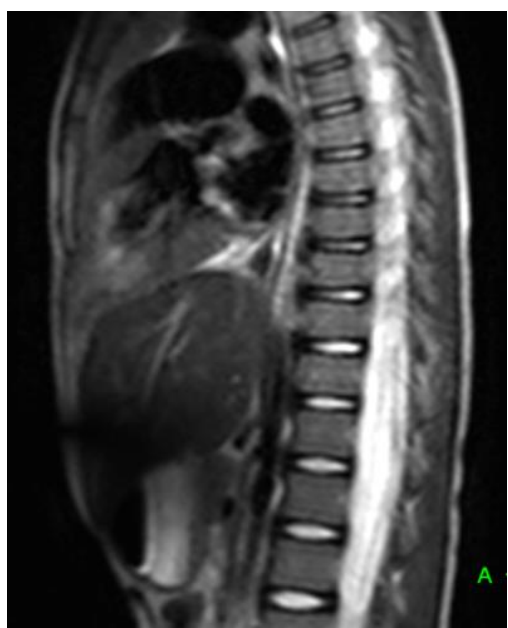

Figure 15 MRI Sagittal plane showing superficial extension of the left ventricle in front of the liver.

A rare congenital anomaly of the heart is described. It consisted of a tube like extension of the left ventricular apex in the middle line toward the umbilicus under the anterior abdominal wall, associated with a membranous subaortic ventricular septal defect and a centrally localized heart in the anterior mediastinum

\section{REFERENCES}

1- Formijne, P. Nederl. Tijdschr. v. Geneesk. (1950). 94, 2704.

2- Snellen, H. A., Dankmeijer, J., Bruin, C., and Collister, R. M. Cardiologia (1952). 21, 563.

3-Clifford Parsons. Ventricular extension into the abdominal wall. Br Heart J. (1957) 19: 34-38. doi: 10.1136/hrt.19.1.34. 